\title{
Functional status and oral health in patients with amyotrophic lateral sclerosis: A cross-sectional study
}

\author{
Alessandro de Sire $^{\mathrm{a}, \mathrm{b}, *}$, Marco Invernizzi $^{\mathrm{a}, \mathrm{c}}$, Martina Ferrillo ${ }^{\mathrm{d}}$, Francesca Gimigliano ${ }^{\mathrm{e}}$, \\ Alessio Baricich $^{\mathrm{a}, \mathrm{c}}$, Carlo Cisari ${ }^{\mathrm{a}, \mathrm{c}}$, Fabiola De Marchi ${ }^{\mathrm{f}}$, Pier Luigi Foglio Bonda ${ }^{\mathrm{g}}$, \\ Letizia Mazzinif $^{\mathrm{f}}$ and Mario Migliariog \\ ${ }^{a}$ Physical and Rehabilitation Medicine, Department of Health Sciences, University of Eastern Piedmont, Novara, \\ Italy \\ ${ }^{\mathrm{b}}$ Rehabilitation Unit, Mons. L. Novarese Hospital, Moncrivello, Vercelli, Italy \\ ${ }^{\mathrm{c}}$ Physical and Rehabilitation Medicine Unit, University Hospital "Maggiore della Caritá", Novara, Italy \\ ${ }^{\mathrm{d}}$ Dental School, Department of Surgical Sciences, University of Turin, Turin, Italy \\ ${ }^{\mathrm{e}}$ Department of Mental and Physical Health and Preventive Medicine, University of Campania Luigi Vanvitelli, \\ Napoli, Italy \\ ${ }^{\mathrm{f}}$ ALS Center, Neurology Unit, University Hospital "Maggiore della Caritá", Novara, Italy \\ ${ }^{\mathrm{g}}$ Dental Clinic, Department of Translational Medicine, University of Eastern Piedmont, Novara, Italy
}

Received 12 August 2020

Accepted 29 October 2020

\begin{abstract}
.
BACKGROUND: Amyotrophic lateral sclerosis (ALS) is a progressive neurodegenerative disease affecting upper and lower motor neurons. The current practice of caring for patients affected by ALS involves a multidisciplinary team without any indication about oral health care.

OBJECTIVE: We sought to investigate the functional status and oral health in patients with ALS to define a specific multidisciplinary management.

METHODS: In this cross-sectional study, we included patients affected by ALS, evaluating their functional status, using the Revised ALS Functional Rating Scale (ALSFRS-R) and their oral health status through specific parameters, including Brief Oral Health Status Examination (BOHSE), Winkel Tongue Coating Index (WTCI), and Oral Food Debris Index (OFDI).

RESULTS: All 37 patients (mean age: $61.19 \pm 11.56$ years) showed a poor oral status, independent from the functional status and strictly correlated to the severity of sialorrhea $(p=0.01)$. OFDI index was negatively correlated with the ALSFRS-R upper limb $(p=0.03)$. Patients with bulbar onset had significantly lower ability to perform adequate tongue movements in terms of protrusion $(p=0.006)$ and lateralization $(p<0.001)$. Significant negative correlations between survival rate and BOHSE $(p=0.03)$ was found.

CONCLUSIONS: Taken together, our findings showed that a poor oral health status might be correlated to a worse functional status and survival time. Thus, an adequate oral health care and rehabilitation should be considered as crucial in the multidisciplinary management of patients with ALS.
\end{abstract}

Keywords: Amyotrophic lateral sclerosis, oral hygiene, functioning, rehabilitation, oral rehabilitation

*Address for correspondence: Alessandro de Sire, MD, Physical and Rehabilitative Medicine, Department of Health Sciences University of Eastern Piedmont "A. Avogadro", Viale Piazza
D’Armi, 1 - 28100 Novara Italy. Tel.: +39 03213734800; E-mail: alessandro.desire@gmail.com. 


\section{Background}

Amyotrophic lateral sclerosis (ALS) is a progressive neurodegenerative disease, caused by motor neuron death in the cerebral motor cortex, brainstem and spinal cord, leading to restricted voluntary movement and detrimental health related quality of life (HRQoL) (Taylor et al., 2016). ALS is characterized by a typical onset in adulthood, with a peak of diagnosis commonly reported from 40 to 70 years old, with 55 years as the mean age of onset (Taylor et al., 2016). The European ALS registries provided an incidence rate of 2.6/100,000 inhabitants/year and a prevalence rate of 7-9/100,000 people (Hardiman et al., 2017), with a mortality occurring within 2-4 years from diagnosis, commonly as a consequence of the respiratory muscle paralysis (Calvo et al., 2020).

ALS might be differentiated according to the region involved at their onset in: spinal onset (SO), associated with signs and symptoms in either the arms, legs, or both and accompanied by lower motor neuron features like muscle atrophy, fasciculations and weakness as well as a combination of pyramidal (upper motor neuron) findings of spasticity and hyperreflexia; bulbar onset (BO), showing dysarthria, dysphagia, and/or dyspnea as the hallmark features (Bergendal \& McAllister, 2017; Easterling et al., 2013).

In this scenario, oral health status is an overlooked issue which is not part of the common clinical management for patients with ALS in most cases. Sialorrhea is one of the most disabling symptoms in ALS, with an increased risk of caries and gum inflammation and could be related to tongue spasticity, orofacial and palatoglossus muscle control failure, facial muscular weakness, as well as the inability to maintain oral and buccal competence (Bergendal \& McAllister, 2017). In literature, a few anecdotical cases have described clinical manifestations like masticatory muscle pain, progressive limitation of mouth opening and macroglossia (Bergendal \& McAllister, 2017; McKee et al., 2013; Pang \& Park, 2015). The worst ALS clinical manifestation is the expiratory muscles weakness, that might lead to pulmonary dysfunction, difficulty in clearing secretions, repeated episodes of pneumonia, and respiratory insufficiency present in almost all cases at some stage of the illness, that might lead to death (Hadjikoutis \& Wiles, 2001; Hardiman, 2011).

The role of rehabilitation in patients affected by ALS at each stage of the disease has been investigated in order to enhance safe mobility and independence, to reduce disease-related impairments and to improve HRQoL (Ferreira et al., 2016; Pinto et al., 2012). However, data about the most effective exercise type, modality and frequency are lacking considering also the need to avoid muscle overuse, according to the disease-related muscular denervation and weakness.

It is assumable that an impairment in oro-facial and upper limbs motor function could prevent an adequate oral health status maintenance; moreover, the decreased tongue mobility could lead to a lower self-cleansing ability and an increased accumulation of food debris and plaque (Bergendal \& McAllister, 2017). In this context, oral health is often limited in individuals with special healthcare needs, with resulting higher rates of dental disorders and increasing disability (Devinsky et al., 2020). Thus, as previously evidenced in stroke survivors (de Sire et al., 2019), a poor oral health status could lead to higher disability, worsening clinical manifestations and HRQoL, and hindering the rehabilitative needs of these subjects.

In light of these considerations, a multidimensional approach that includes neurologists, physical medicine and rehabilitation (PRM) physicians, and oral care specialists is strongly recommended for patients affected by ALS (Miller et al., 2009; Norris et al., 2020; Park D, et al., 2020; Plowman et al., 2019); however, oral hygiene screening is mandatory in these patients. However, albeit the current practice takes into account a multidisciplinary team, there is still a lack of clear indications regarding oral health care and rehabilitation needs patients with ALS.

Therefore, aim of the present study was to investigate functional status and oral health in a sample of patients with ALS, according to their onset; furthermore, we evaluated the correlation among oral health status and clinical manifestations and survival time, in order to provide evidence on the importance of a patient-oriented multidisciplinary management.

\section{Methods}

\subsection{Participants}

In this cross-sectional study, we recruited adult patients with ALS referred to the Tertiary ALS Center of University Hospital 'Maggiore della Caritá' of Novara, Italy, over a 12-month period from January to December 2019.

Inclusion criteria were: 1) a diagnosis of clinically definite, probable, probable laboratory-supported or possible ALS according to 'El Escorial Revisited: 
revised criteria' (Brooks et al., 2000); 2) have attended at least one appointment at the multidisciplinary ALS clinic; 3) being able to provide informed consent in person or through a legally authorized representative. Exclusion criteria were: 1) psychiatric diseases or neurological diseases other than ALS; 2) severe mental deterioration which makes it impossible to understand orders; 3 ) evidences of concurrent illness; 4) undergoing treatment with corticosteroids, immunoglobulin, or immunosuppressive drugs; 5) less than 10 teeth; 6 ) suffering or having suffered in the past from major oral infections; 7) unable to understand the informed consent.

The study was approved by the Local Ethics Committee (CE 61/10, prot.392 CE). All participants were asked to carefully read and sign an informed consent, and researchers provided to protect the privacy and the study procedures according to the Declaration of Helsinki, with pertinent National and International regulatory requirements. Moreover, the study was performed in accordance with the STrengthening the Reporting of OBservational studies in Epidemiology (STROBE) Guidelines.

\subsection{Intervention}

At the admission to the hospital, PRM physicians assessed the functional status of all study participants and propose a patient-oriented rehabilitation treatment to prevent potential ALS-related complications. Furthermore, patients also underwent a clinical assessment of dental formula (including the presence of filled or decayed teeth) and oral health status by oral care specialists.

\subsection{Outcome measures}

For all patients included in the study we collected the following data at the admission to the hospital: demographic characteristics; disease duration; type of onset (SO or $\mathrm{BO}$ ); Force Vital Capacity percentage (FVC\%); Body Mass Index (BMI); feeding status (autonomous, assisted or undergoing percutaneous endoscopic gastrostomy - PEG); dysphagia score using Strand Scale (Strand et al., 1996); type of diet; use of Non-Invasive Ventilation (NIV) with number of hours/day and months of use; sialorrhea; social cognitive impairment, defined according to Strong Criteria (Strong et al., 2017); survival time, calculated as the time from symptoms onset and death or tracheostomy (for living patients we used as censored date April 2020).
Revised Amyotrophic Lateral Sclerosis Functional Rating Scale (ALSFRS-R) was used to assess the functional status, considering also its sub-scores for upper and lower limbs, respiratory functions and bulbar functions (Gordon et al., 2004).

Furthermore, we evaluated oral health status, trough the following outcome measures: ability to perform tongue protrusion, and right and left lateralization; Kayser-Jones Brief Oral Health Status Examination (BOHSE) (Kayser-Jones et al., 1995) to assess the global oral health status; Winkel Tongue Coating Index (WTCI) (Winkel et al., 2003) to evaluate the amount of tongue coating; New Method of Plaque Scoring (NMPS) (Dababneh et al. 2002) to assess the presence of plaque on the teeth; Gingival Index (GI) (Löe \& Silness, 1963) to evaluate the signs of gingival inflammation; Oral Hygiene Index (OHI) (Greene \& Vermillon, 1964) to assess the presence of debris/stain and tartar on the teeth; Oral Food Debris Index (OFDI) (Migliario \& Rimondini, 2011) to evaluate the food debris in the oral cavity.

\subsection{Statistical analysis}

Data management and analyses were conducted according to a pre-specified statistical analytical plan. Statistical analysis was performed using STATA v. 12 (StataCorp LP, College Station, TX, USA). The continuous variables are presented as means \pm standard deviations, or median and interquartile range. The Shapiro-Wilk test was performed to assess the distribution of all continuous data; as the data did not follow a normal distribution, Wilcoxon rank sum test was used to compare continuous variables between the two groups; Fisher exact test was performed to compare categorical variables between the two groups. Pearson correlation coefficients and regression analyses assessed associations and correlations among oral health status of study participants, analyzing a correlation with clinical and demographic features. A $p$-value of 0.05 was considered statistically significant.

\section{Results}

\subsection{Demographic and clinical characteristics}

Out of 51 patients affected by ALS recruited, 37 (12 men and 25 women), mean aged $61.19 \pm 11.56$ years, were included for the final analysis. Nine were excluded because having less than 10 teeth, and 5 
Table 1

Study population characteristics

\begin{tabular}{ll}
\hline & Total $(n=37)$ \\
\hline Age (years) & $61.19(11.56)$ \\
Sex (male/female) & $12 / 25$ \\
Disease duration at the & $20(8-41)$ \\
$\quad$ admission (months) & \\
Survival time (months) & $43(29.5-70.75)$ \\
BMI & $23.37(4.45)$ \\
FVC\% & $64.33821 .98)$ \\
NIV (bulbar/spinal) & $4 / 5$ \\
Mean NIV hours/day & $8.00(3.92)$ \\
Mean months of use & $12.55(9.80)$ \\
Sialorrhea (bulbar/spinal) & $7 / 10$ \\
Treatment for & 9 \\
$\quad$ sialorrhea (number) & \\
PEG (bulbar/spinal) & $1 / 0$ \\
Cognitive status & $25 / 12$ \\
$\quad$ normal/deficit) & \\
ALSFRS-R score & $28.27(7.80)$ \\
ALSFRS- $R$ bulbar & $7.57(2.97)$ \\
ALSFRS- $R$ UL & $5.35(3.78)$ \\
ALSFRS- $R$ LL & $5.81(3.83)$ \\
ALSFRS- $R$ respiratory & $9.54(2.62)$ \\
BOHSE & $4.54(2.37)$ \\
WTCI & $1.18(0.55)$ \\
NMPS & $4.39(2.65)$ \\
GI & $1.32(0.81)$ \\
OHI & $1.95(1.33)$ \\
OFDI & $0.62(0.59)$ \\
\hline Continyou vatiable are &
\end{tabular}

Continuous variables are expressed as means (standard deviations) or medians (interquartile ranges); ratios are expressed as $\mathrm{x} / \mathrm{y}$. Abbreviations: ALS = amyotrophic lateral sclerosis; ALSFRS-R: ALS Functional Rating Scale - revised; BMI: Body Mass Index; BOHSE $=$ Kayser-Jones Brief Oral Health Status Examination; FVC: Force Vital Capacity; GI = Gingival Index; LL: lower limb; NIV = Non-Invasive Ventilation; NMPS = New Method of Plaque Scoring; Oral Food Debris Index (OFDI); OHI=Oral Hygiene Index; $\mathrm{PEG}=$ percutaneous endoscopic gastrostomy; UP: upper limb; WTCI $=$ Winkel Tongue Coating Index.

as their diagnosis of ALS was not confirmed. In our cohort, one patient had PEG. Demographic and clinical characteristics are summarized in Table 1.

\subsection{Functional and oral health status and type of ALS onset}

Patients were divided according to the onset of ALS into 2 groups: BO group $(n=10$; mean age: $62.5 \pm 8.2$ years) and SO group $(n=27$; mean age: $60.7 \pm 1.7$ years).

There were no significant differences between groups in terms of age, age at ALS diagnosis, and autonomous feeding. However, number of patients undergoing assisted feeding was significantly higher in SO patients compared to those with $\mathrm{BO}(p=0.01)$. As depicted by Table 2, the SO group had significantly lower levels than $\mathrm{BO}$ group in terms of
ALSFRS-R total $(26.3 \pm 7.6$ vs $33.7 \pm 5.5 ; p=0.01)$, and ALSFRS-R UL $(p<0.01)$ and ALSFRS-R LL $(p<0.01)$ sub-scores. On the contrary, BO group had significantly lower ALSFRS-R bulbar sub-score $(p=0.04)$. There were no differences between groups $(p=0.54)$ in ALSFRS-R respiratory sub-score.

BO patients had a significantly lower ability than SO patients in terms of tongue protrusion $(40.0 \%$ vs $85.2 \% ; p<0.01)$, right lateralization $(30.0 \%$ vs $88.9 \% ; p<0.001)$, and left lateralization $(30.0 \%$ vs $85.2 \% ; p<0.01)$. There was any significant difference between subjects with $\mathrm{SO}$ and $\mathrm{BO}$ in terms of oral health status indexes (see Table 2 for further details).

\subsection{Oral health status and correlations with clinical features}

The BOHSE showed an impairment of oral health status in 7 out 37 patients (18.9\%). Higher scores strongly correlated with presence of sialorrhea $(p=$ 0.01 ), independently by concomitant symptomatic ongoing treatment (see Fig. 1, left). A significant correlation was observed between sialorrhea and WTCI $(p=0.04)$. A positive trend was found also between sialorrhea and gingival index $(p=0.06)$.

We found a positive correlation between undergoing NIV through nasal mask and oral health status, assessed by BOHSE $(p=0.03)$ (see Fig. 1, right); there was also a positive trend for $\mathrm{OHI}$ and GI $(p=0.06)$.

The OFDI index was negatively correlated with the total ALSFRS-R $(R=-0.40 ; p=0.04)$, mainly with the upper limb score $(R=-0.40 ; p=0.03)$.

Lastly, no significant correlations $(p>0.05)$ were observed between all oral health indexes and the presence of social cognitive impairment, assessed according to Strong Criteria (Strong et al., 2017).

\subsection{Oral health status and correlations with dysphagia, BMI and type of diet}

Level of dysphagia was positively correlated with WTCI $(R=0.37 ; p=0.02)$. No other significant correlations were observed between oral health status indexes and dysphagia. Similarly, no significant correlations were found between oral health status indexes and both BMI and type of diet $(p>0.05)$.

\subsection{Oral health status and survival time}

A median survival time in this cohort from onset to death or tracheostomy was 43 months (IQR: 
Table 2

Differences between bulbar onset and spinal onset in patients with ALS

\begin{tabular}{|c|c|c|c|c|}
\hline & $\begin{array}{c}\text { Total } \\
(n=37)\end{array}$ & $\begin{array}{c}\text { ALS bulbar } \\
\text { onset }(n=10)\end{array}$ & $\begin{array}{c}\text { ALS spinal } \\
\text { onset }(n=27)\end{array}$ & $p$-value \\
\hline$\overline{\text { Age (years) }}$ & $61.19 \pm 11.56$ & $62.54 \pm 8.23$ & $60.69 \pm 1.67$ & $0.67^{*}$ \\
\hline Age at ALS diagnosis (years) & $57.70 \pm 13.24$ & $61.24 \pm 8.33$ & $56.40 \pm 14.57$ & $0.33^{*}$ \\
\hline Sex (male/female) & $12 / 25$ & $4 / 6$ & $8 / 19$ & $0.55^{* *}$ \\
\hline Autonomous feeding & $17(45.95 \%)$ & $7(70.00 \%)$ & $10(37.04 \%)$ & $0.14^{* *}$ \\
\hline Assisted feeding & $18(48.65 \%)$ & $1(10.00 \%)$ & $17(62.96 \%)$ & $0.01^{* *}$ \\
\hline Tongue protrusion & $27(72.97 \%)$ & $4(40.00 \%)$ & $23(85.19 \%)$ & $0.01^{*}$ \\
\hline Tongue right lateralization & $27(72.97 \%)$ & $3(30.00 \%)$ & $24(88.89 \%)$ & $<0.01^{*}$ \\
\hline Tongue left lateralization & $26(70.27 \%)$ & $3(30.00 \%)$ & $23(85.19 \%)$ & $0.01^{*}$ \\
\hline ALSFRS-R total & $28.27 \pm 7.81$ & $33.70 \pm 5.49$ & $26.26 \pm 7.63$ & $0.01^{*}$ \\
\hline ALSFRS-R bulbar & $7.57 \pm 2.97$ & $5.90 \pm 5.49$ & $8.19 \pm 2.98$ & $0.04^{*}$ \\
\hline$A L S F R S-R U L$ & $5.35 \pm 3.79$ & $8.80 \pm 5.49$ & $4.07 \pm 3.03$ & $<0.01^{*}$ \\
\hline$A L S F R S-R L L$ & $5.81 \pm 3.84$ & $9.90 \pm 2.72$ & $4.30 \pm 2.99$ & $<0.01^{*}$ \\
\hline$A L S F R S-R$ respiratory & $9.54 \pm 2.62$ & $9.10 \pm 2.46$ & $9.73 \pm 2.70$ & $0.54^{*}$ \\
\hline BOHSE & $4.54 \pm 2.37$ & $4.10 \pm 1.72$ & $4.70 \pm 2.58$ & $0.50^{* *}$ \\
\hline WTCI & $1.18 \pm 0.55$ & $1.43 \pm 0.53$ & $1.09 \pm 0.54$ & $0.23^{* *}$ \\
\hline NMPS & $4.39 \pm 2.65$ & $4.04 \pm 1.19$ & $4.51 \pm 3.03$ & $0.64^{* *}$ \\
\hline GI & $1.32 \pm 0.81$ & $1.16 \pm 0.53$ & $1.38 \pm 0.90$ & $0.47^{* *}$ \\
\hline $\mathrm{OHI}$ & $1.95 \pm 1.33$ & $1.50 \pm 0.83$ & $2.12 \pm 1.45$ & $0.29^{* *}$ \\
\hline OFDI & $0.62 \pm 0.59$ & $0.63 \pm 0.57$ & $0.60 \pm 0.70$ & $0.77^{* *}$ \\
\hline
\end{tabular}

Continuous variables are expressed as means \pm standard deviations; categorical variables are expressed as counts (percentages). Statistical analysis tests performed were: ${ }^{*}=$ Wilcoxon rank sum test; ${ }^{* *}=$ Fisher exact test; A $p$-value of 0.05 was considered statistically significant. Abbreviations: ALS = amyotrophic lateral sclerosis; ALSFRS-R=ALS Functional Rating Scale - revised; BOHSE = Kayser-Jones Brief Oral Health Status Examination; GI = Gingival Index; LL = lower limbs; NMPS = New Method of Plaque Scoring; OHI = Oral Hygiene Index; Oral Food Debris Index = OFDI; UL = upper limbs; WTCI = Winkel Tongue Coating Index.
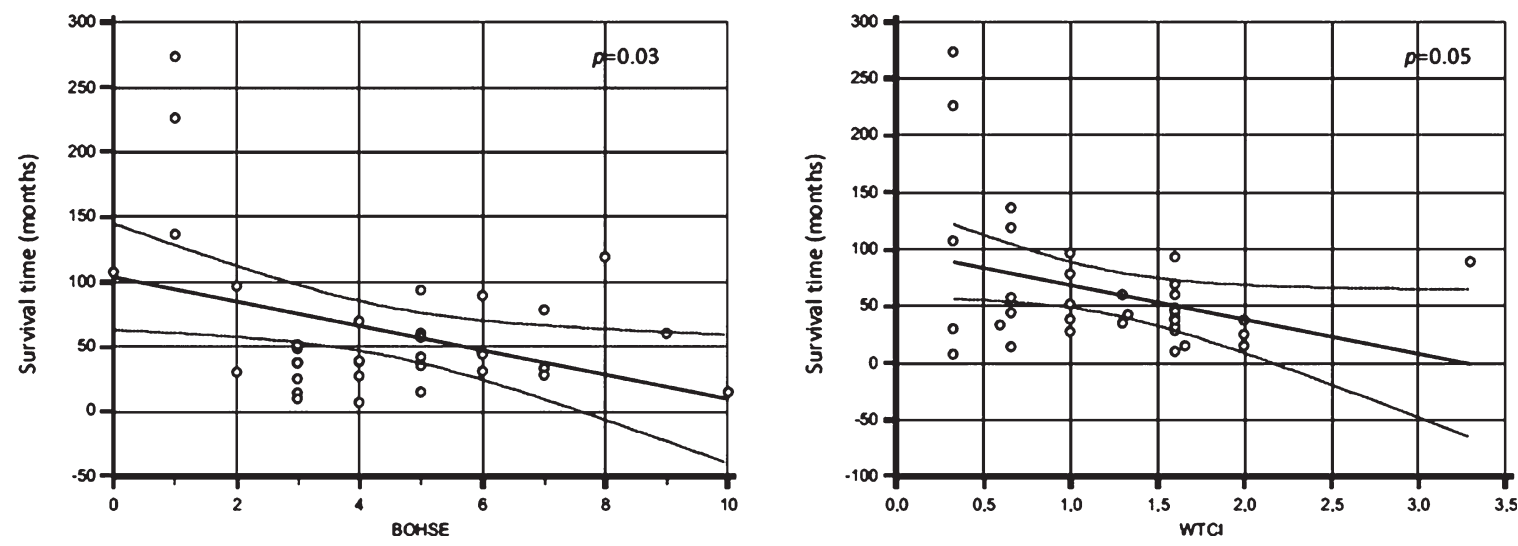

Fig. 1. Correlation between Kayser-Jones Brief Oral Health Status Examination (BOHSE) and sialorrhea $(p=0.01)($ left $)$ and Non-Invasive Ventilation (NIV) $(p=0.03)$ (right).

29.5-70.75). At the censoring date, 22 patients were dead or with tracheostomy.

Firstly, BOHSE, WTCI, GI, OFDI, and OHI were significantly correlated with survival time showing the oral health status could influence ALS duration. Pearson correlation analysis showed a negative correlation between survival rate and BOHSE $(R=-0.40$; $p=0.03)$ (see Fig. 2, left) and WTCI $(R=-0.35 ; p=$ $0.05)$ (see Fig. 2, right). A multiple linear regression model, including BOHSE, WTCI, GI, OFDI, OHI, age, and sex, showed a significant correlation between survival time and WTCI $(p=0.02)$ and between survival time and age $(p=0.009)$.

\section{Discussion}

To the best of our knowledge, this is the first study that focused on functional status and oral health in patients affected by ALS in the context of a multidisciplinary care approach. The most important result of this study is the demonstration that most 

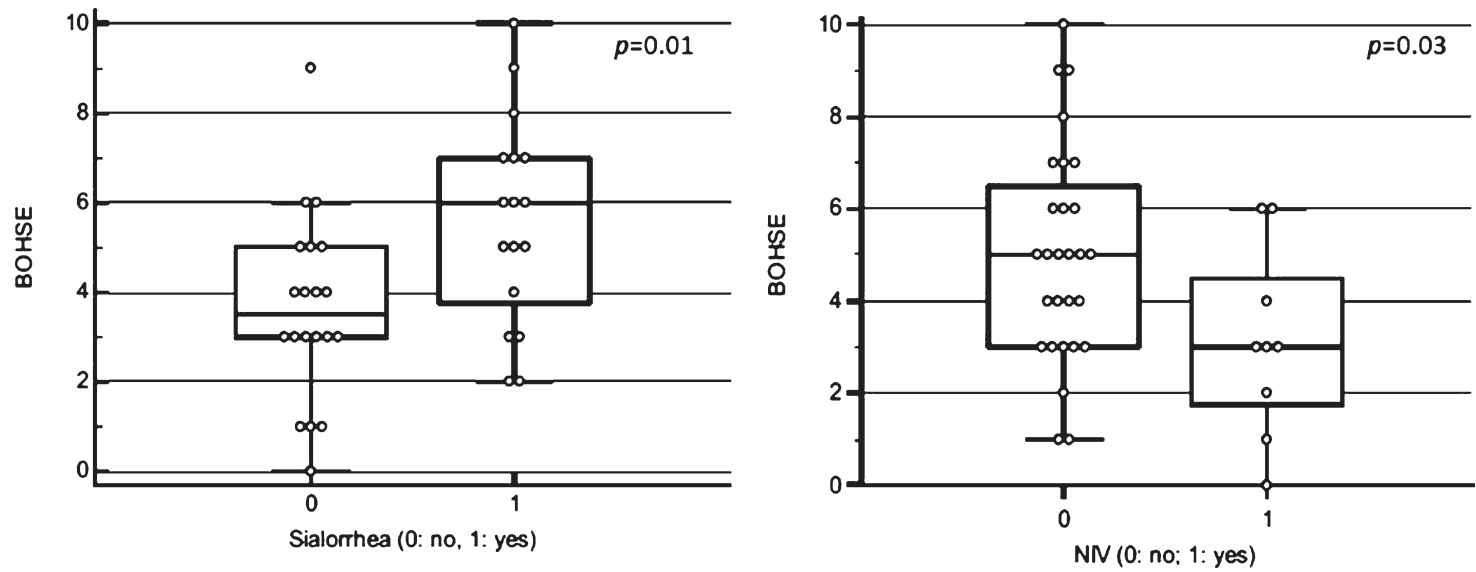

Fig. 2. Correlation between survival time (months) and Kayser-Jones Brief Oral Health Status Examination (BOHSE) $(p=0.03)($ left) and Winkel Tongue Coating Index (WTCI) $(p=0.05)$ (right).

of them showed a poor oral status, which was independent from the functional status and was strictly correlated with a poor survival.

In this cross-sectional study, we showed that total ALSFRS-R score was significantly lower $(p=0.008)$ in SO patients compared to BO, However, considering each ALSFRS-R sub-item we found worse results only in ALSFRS-R UL $(p<0.001)$ and ALSFRS-R LL $(p<0.001)$ in the SO group.

A global poor oral status in patients with ALS was detailed in terms of oral health (evaluated by BOHSE), amount of tongue coating (assessed by WTCI), presence of plaque on the teeth (as showed by NMPS), gingival inflammation (assessed by GI score) and presence of debris/stain and tartar on the teeth (evaluated by $\mathrm{OHI}$ ).

In the context of clinical parameters, we observed a positive correlation between sialorrhea and BOHSE, WTCI and GI, suggesting as an excess of salivation is an invalidating symptom and can cause a range of clinical and functional complications such as aspiration, bad odor and infection.

Moreover, as expected, patients with ALS with BO had a significantly worse lingual mobility than SO patients in terms of protrusion and lateralization. A prospective case-series study previously investigated both functional status and oral health in patients with ALS according to their site of onset in 14 ALS patients (Bergendal \& McAllister, 2017). The patients affected by ALS with BO $(n=8)$ had more severe orofacial impairment compared to those with SO $(n=6)$, as showed by Nordic Orofacial TestScreening (NOT-S) (5.6 vs. 0.7 at initial registration; 6.1 vs. 3.2 at final registration). These data are in line with our results, although performed on a case-series design and using a different instrumental tool (NOT-S vs. ALSFRS-R for orofacial functions evaluation) in two different time-points (before and after the introduction of oral and dental aids). It is also shown that patients affected by ALS with BO had severe impairment in orofacial functions from an early disease stage, confirming the disease main features. Moreover, the Authors concluded that all patients with ALS independently from the type of early stage symptoms needed assistance in performing oral hygiene measures. This notion is in line also with a cross-sectional study performed by Nakayama et al. (Nakayama et al., 2018) aimed to analyze oral condition and function in hospitalized patients affected by ALS treated by tracheostomy positive-pressure ventilation. The oral cavity of all patients was cleaned at least twice per day, including the removal of accumulated liquid in the mouth by aspiration, intratracheal aspiration, tongue care and teeth cleaning. The Authors concluded that severe dental disease is quite uncommon among hospitalized patients with ALS independently from their onset and is related to the oral care that they could receive, suggesting the need to sensitize caregivers in the outpatient setting about these potential issues. Abe et al. (Abe et al., 2008) pointed out how an adequate oral hygiene could reduce oral plaque and help reduce the risk for aspiration pneumonia in patients affected by ALS. Indeed, aspiration of oral bacteria due to swallowing impairment and cough reflexes associated with cerebrovascular disorders has been reported to be a common cause of aspiration pneumonia (Bartlett et al., 1974). Inglis et al. (Inglis et al., 1993) reported that the main 
determinant in the pathogenesis of aspiration pneumonia was not the type of oral bacteria but the total amount of bacteria aspirated. Therefore, considering that the tongue has been reported to be a large reservoir of oral bacteria, oral health status and impairments should be adequately evaluated and treated in order to prevent aspiration pneumonia, a frequent and well-known cause of death in patients with ALS (Sumi et al., 2006).

Furthermore, a negative trend is highlighted between survival and both BOHSE and WTCI, suggesting as the oral health status can be included as indicator of general health.

Lastly, it should be underlined that the oral health status was poor in all study participants, suggesting that an incorrect oral hygiene may be more related to lower functional status, testifying the importance of rehabilitation in patients with ALS.

Why do patients with ALS need rehabilitation? Firstly, the poor functional status evidenced by ALSFRS-R testified that an appropriate rehabilitative intervention should be prescribed by a PRM physician in order to counteract muscle impairment. A recent meta-analysis performed by Park et al. demonstrated that therapeutic exercise exerted benefic effects in patients with ALS in terms of preventing the progression of limb weakness and improving cardiopulmonary benefit (Park et al., 2020).

Furthermore, it is well known that ALS is characterized by motor neurons degeneration, leading to the loss of muscle mass and atrophy with progressive motor impairment, including ventilation insufficiency (Pinto et al., 2012). Respiratory muscles weakness could impair airway protection by reducing the expiratory pressure generation capacities necessary to produce an effective cough (Ruoppolo et al., 2013). Expiratory muscles weakness is a widespread condition in patients affected by advanced neurodegenerative diseases and could result in pulmonary dysfunction and consequent pneumonia, one of the main causes of death in patients with ALS (Hadjikoutis \& Wiles, 2001; Hardiman, 2011). Expiratory muscle strength training was demonstrated to be welltolerated and effective in improving respiratory and bulbar function in patients with ALS (Plowman et al., 2019).

It is well known that NIV might compensate diaphragm weakness, leading to a prolonged survival and higher HRQoL in patients affected by ALS with clinically significant expiratory muscle weakness ( $\mathrm{Ng}$ et al., 2017). Although NIV therapy is more complicated in $\mathrm{BO}$ patients because of hypersalivation and oral functional impairments, Berlowitz et al. demonstrated that BO patients undergoing NIV therapy might have a prolonged survival rate comparable to those observed in SO patients (Berlowitz et al., 2016). Therefore, in light of its non-invasiveness and adequate tolerability, NIV could be considered as an essential therapeutic component in the rehabilitative management of patients with ALS with both onsets and a prompt and effective oral health evaluation and treatment could facilitate its use, in particular in those with $\mathrm{BO}$.

It was interesting to notice in our cohort that patients using NIV device through nasal mask showed to have a positive correlation with oral health status, assessed by BOHSE $(p=0.03)$; these findings might be due to the lower presence of saliva. However, taking into account the small number of patients using NIV in our cohort (24.3\%) the impact of ventilation on oral health status should be better investigated in future studies on a wider sample.

Thus, as previously evidenced by our group in stroke survivors (de Sire et al., 2019), oral care screening should be implemented in the routine clinical management of neurological patients, to define prompt and effective oral rehabilitation intervention.

Lastly, given the complex and multifaceted clinical scenario and sequelae of ALS, the International Classification of Functioning, Disability and Health (ICF) (Cieza et al., 2004), taking into account impairments, activity limitations, and participation restrictions, might lead to a better overview of the overall functional status of patients with ALS (Ciotti et al., 2018). To date, patients affected by ALS might be assessed by ICF Generic and Rehabilitation Sets, minimal sets of ICF categories applied for ICFbased reporting in the clinical practice (Prodinger et al., 2016; Gimigliano et al., 2019). In the future, ICF could become a keystone in the comprehensive and multidisciplinary management of patients with ALS in order to set up more effective and personalized rehabilitation programs (Ciotti et al., 2018).

This study has some limitations: first, in our cohort, the patients affected by ALS with BO are fewer compared to SO group, clearly reflecting the epidemiology of the disease and can be explained by the real-practice nature of the study, but it could be generating a bias in the evaluation; second, the cross-sectional study design hinders any potential assessment about the impact of a rehabilitation intervention in modifying the functional outcomes. 
However, as previously noticed, to the best of our knowledge, this is the first cross-sectional study that investigated functional status, tongue mobility, and oral health in patients affected by ALS with SO and BO. After these preliminary results, our goal is continuing the oral assessment evaluation both in clinical practice and in research setting, associating a prompt and patient-tailored rehabilitation program to improve the oral functional outcomes and their management.

\section{Conclusions}

Taken together, our findings corroborate the hypothesis that a poor oral health status could be correlated to a worse functional status and survival time, making necessary the role of an adequate oral rehabilitation management in patients with ALS.

Thus, we suggest including oral care and rehabilitation in the comprehensive and multidisciplinary management of patients affected by ALS in order to improve functioning, HRQoL, and survival time. Further prospective studies are warranted to confirm these data and to clarify the role of a patientoriented rehabilitative intervention in patients with ALS according to their onset.

\section{Conflict of interest}

Nothing to declare.

\section{Funding}

None to report.

\section{References}

Abe, S., Ishihara, K., Adachi, M., \& Okuda, K. (2008). Tonguecoating as risk indicator for aspiration pneumonia in edentate elderly. Archives of Gerontology and Geriatrics, 47(2), 267275.

Bartlett, J. G., Gorbach, S. L., \& Finegold, S. M. (1974). The bacteriology of aspiration pneumonia. The American Journal of Medicine, 56(2), 202-207.

Bergendal, B., \& McAllister, A. (2017). Orofacial function and monitoring of oral care in amyotrophic lateral sclerosis. Acta Odontologica Scandinavica, 75(3), 179-185.

Berlowitz, D. J., Howard, M. E., Fiore, J. F., Jr, Vander Hoorn, S., O’Donoghue, F. J., Westlake, J., Smith, A., Beer, F., Mathers, S., \& Talman, P. (2016). Identifying who will benefit from non-invasive ventilation in amyotrophic lateral sclerosis/motor neurone disease in a clinical cohort. Journal of Neurology, Neurosurgery, and Psychiatry, 87(3), 280-286.

Brooks, B. R., Miller, R. G., Swash, M., Munsat, T. L., \& World Federation of Neurology Research Group on Motor Neuron Diseases (2000). El Escorial revisited: revised criteria for the diagnosis of amyotrophic lateral sclerosis. Amyotrophic lateral sclerosis and other motor neuron disorders: official publication of the World Federation of Neurology, Research Group on Motor Neuron Diseases, 1(5), 293-299.

Calvo, A., Vasta, R., Moglia, C., Matteoni, E., Canosa, A., Mattei, A., et al. (2020). Prognostic role of slow vital capacity in amyotrophic lateral sclerosis. Journal of Neurology, 267(6), 1615-1621.

Cieza, A., Stucki, G., Weigl, M., Disler, P., Jäckel, W., van der Linden, S., Kostanjsek, N., \& de Bie, R. (2004). ICF Core Sets for low back pain. Journal of Rehabilitation Medicine, (44 Suppl), 69-74.

Ciotti, S., Bianconi, F., Saraceni, V. M., Vulpiani, M. C., Rinonapoli, G., Caraffa, A., \& Zampolini, M. (2018). Narrative Medicine in Amyotrophic Lateral Sclerosis and a Rehabilitation Project Based on International Classification of Functioning, Disability and Health. American Journal of Physical Medicine \& Rehabilitation, 97(11), 832-838.

Dababneh, R. H., Khouri, A. T., Smith, R. G., \& Addy, M. (2002). Correlation and examiner agreement between a new method of plaque scoring and a popular established plaque index, modelled in vitro. Journal of Clinical Periodontology, 29(12), 1107-1111.

de Sire, A., Baricich, A., Ferrillo, M., Migliario, M., Cisari, C., \& Invernizzi, M. (2020). Buccal hemineglect: is it useful to evaluate the differences between the two halves of the oral cavity for the multidisciplinary rehabilitative management of right brain stroke survivors? A cross-sectional study. Topics in Stroke Rehabilitation, 27(3), 208-214.

Devinsky, O., Boyce, D., Robbins, M., \& Pressler, M. (2020). Dental health in persons with disability. Epilepsy \& Behaviour, 110, 107174.

Easterling, C., Antinoja, J., Cashin, S., \& Barkhaus, P. E. (2013). Changes in tongue pressure, pulmonary function, and salivary flow in patients with amyotrophic lateral sclerosis. Dysphagia, 28(2), 217-225.

Ferreira, G. D., Costa, A. C., Plentz, R. D., Coronel, C. C., \& Sbruzzi, G. (2016). Respiratory training improved ventilatory function and respiratory muscle strength in patients with multiple sclerosis and lateral amyotrophic sclerosis: systematic review and meta-analysis. Physiotherapy, 102(3), 221-228.

Gimigliano, F., De Sire, A., Gastaldo, M., Maghini, I., Paoletta, M., Pasquini, A., Boldrini, P., Selb, M., Prodinger, B., \& SIMFER Residents Section Group (2019). Use of the International Classification of Functioning, Disability and Health Generic-30 Set for the characterization of outpatients: Italian Society of Physical and Rehabilitative Medicine Residents Section Project. European Journal of Physical and Rehabilitation Medicine, 55(2), 258-264.

Gordon, P. H., Miller, R. G., \& Moore, D. H. (2004). ALSFRS-R. Amyotrophic lateral sclerosis and other motor neuron disorders: official publication of the World Federation of Neurology, Research Group on Motor Neuron Diseases, 5 (Suppl 1), 90-93.

Greene J.C., Vermillon J.R. (1964) The Simplified Oral Hygiene Index. Journal of the American Dental Association, 68, 7-13. 
Hadjikoutis, S., \& Wiles, C.M. (2001). Respiratory complications related to bulbar dysfunction in motor neuron disease. Acta Neurologica Scandinavica, 103(4), 207-213.

Hardiman, O. (2011). Management of respiratory symptoms in ALS. Journal of Neurology, 258(3), 359-365.

Hardiman, O., Al-Chalabi, A., Brayne, C., Beghi, E., van den Berg, L. H., Chio A., et al. (2017). The changing picture of amyotrophic lateral sclerosis: lessons from European registers. Journal of Neurology, Neurosurgery, and Psychiatry, 88(7), 557-563.

Inglis, T. J., Sherratt, M. J., Sproat, L. J., Gibson, J. S., \& Hawkey, P. M. (1993). Gastroduodenal dysfunction and bacterial colonisation of the ventilated lung. Lancet (London, England), 341(8850), 911-913.

Kayser-Jones, J., Bird, W. F., Paul, S. M., Long, L., \& Schell, E. S. (1995). An instrument to assess the oral health status of nursing home residents. The Gerontologist, 35(6), 814-824.

Löe H., Silness J. (1963) Periodontal disease in pregnancy. I. Prevalence and severity. Acta odontologica Scandinavica, 21, 533-51.

McKee, H. R., Escott, E., Damm, D., \& Kasarskis, E. (2013). Macroglossia in amyotrophic lateral sclerosis. JAMA Neurology, 70(11), 1432-1435.

Migliario, M., \& Rimondini, L. (2011). Food debris index. British Dental Journal, 211(12), 581-582.

Miller, R. G., Jackson, C. E., Kasarskis, E. J., England, J. D., Forshew, D., Johnston, W., et al., \& Quality Standards Subcommittee of the American Academy of Neurology (2009). Practice parameter update: the care of the patient with amyotrophic lateral sclerosis: drug, nutritional, and respiratory therapies (an evidence-based review): report of the Quality Standards Subcommittee of the American Academy of Neurology. Neurology, 73(15), 1218-1226.

Nakayama, R., Nishiyama, A., Matsuda, C., Nakayama, Y., Hakuta, C., \& Shimada, M. (2018). Oral health status of hospitalized amyotrophic lateral sclerosis patients: a single-centre observational study. Acta odontologica Scandinavica, 76(4), 294-298.

Ng, L., Khan, F., Young, C. A., \& Galea, M. (2017). Symptomatic treatments for amyotrophic lateral sclerosis/motor neuron disease. The Cochrane database of systematic reviews, 1(1), CD011776.

Norris, S.P., Likanje, M.N., \& Andrews, J.A. (2020). Amyotrophic lateral sclerosis: update on clinical management. Current Opinion in Neurology, 33(5), 641-648.

Pang, K.M., \& Park, J.W. (2015). Masticatory muscle pain and progressive mouth opening limitation caused by amyotrophic lateral sclerosis: a case report. J Oral Facial Pain Headache, 29(1), 91-96.
Park, D., Kwak, S.G., Park, J.S., Choo, Y.J., \& Chang, M.C. (2020). Can Therapeutic Exercise Slow Down Progressive Functional Decline in Patients With Amyotrophic Lateral Sclerosis? A Meta-Analysis. Frontiers in Neurology, 11, 853.

Pinto, S., Swash, M., \& de Carvalho, M. (2012). Respiratory exercise in amyotrophic lateral sclerosis. Amyotrophic lateral sclerosis: official publication of the World Federation of Neurology Research Group on Motor Neuron Diseases, 13(1), 33-43.

Plowman, E. K., Tabor-Gray, L., Rosado, K. M., Vasilopoulos, T., Robison, R., Chapin, J. L., et al. (2019). Impact of expiratory strength training in amyotrophic lateral sclerosis: Results of a randomized, sham-controlled trial. Muscle \& Nerve, 59(1), 40-46.

Prodinger, B., Cieza, A., Oberhauser, C., Bickenbach, J., Üstün, T. B., Chatterji, S., \& Stucki, G. (2016). Toward the International Classification of Functioning, Disability and Health (ICF) Rehabilitation Set: A Minimal Generic Set of Domains for Rehabilitation as a Health Strategy. Archives of Physical Medicine and Rehabilitation, 97(6), 875-884.

Ruoppolo, G., Schettino, I., Frasca, V., Giacomelli, E., Prosperini, L., Cambieri, C., Roma, R., Greco, A., Mancini, P., De Vincentiis, M., Silani, V., \& Inghilleri, M. (2013). Dysphagia in amyotrophic lateral sclerosis: prevalence and clinical findings. Acta neurologica Scandinavica, 128(6), 397-401.

Strand, E. A., Miller, R. M., Yorkston, K. M., \& Hillel, A. D. (1996). Management of oral-pharyngeal dysphagia symptoms in amyotrophic lateral sclerosis. Dysphagia, 11(2), 129-139.

Strong, M. J., Abrahams, S., Goldstein, L. H., Woolley, S., Mclaughlin, P., Snowden, J., Mioshi, E., Roberts-South, A., Benatar, M., HortobáGyi, T., Rosenfeld, J., Silani, V., Ince, P. G., \& Turner, M. R. (2017). Amyotrophic lateral sclerosis - frontotemporal spectrum disorder (ALS-FTSD): Revised diagnostic criteria. Amyotrophic Lateral Sclerosis \& Frontotemporal Degeneration, 18(3-4), 153-174.

Sumi, Y., Miura, H., Nagaya, M., Michiwaki, Y., \& Uematsu, H. (2006). Colonisation on the tongue surface by respiratory pathogens in residents of a nursing home-a pilot study. Gerodontology, 23(1), 55-59.

Taylor, J. P., Brown, R. H. Jr, \& Cleveland, D. W. (2016). Decoding ALS: from genes to mechanism. Nature, 539(7628), 197-206.

Winkel, E. G., Roldán, S., Van Winkelhoff, A. J., Herrera, D., \& Sanz, M. (2003). Clinical effects of a new mouthrinse containing chlorhexidine, cetylpyridinium chloride and zinc-lactate on oral halitosis. A dual-center, double-blind placebo-controlled study. Journal of clinical periodontology, 30(4), 300-306. 